\title{
Interactive Effects of Nitrogen and Humic Substances Applications on Bioethanol Production from Sweet Sorghum and Combustion Characteristics of Its Bagasse
}

\author{
Recep Irfan Nazli ${ }^{1, *}$, Veyis Tansi ${ }^{1}$, Osman Gulnaz ${ }^{2}$, Ebru Kafkas ${ }^{3}$, Alpaslan Kusvuran ${ }^{4}$, \\ Hasan Huseyin Ozturk ${ }^{5}$ and Dilek Bostan Budak ${ }^{6}$ \\ 1 Department of Field Crops, Faculty of Agriculture, Cukurova University, Adana 01330, Turkey; \\ veyis@cu.edu.tr \\ 2 Department of Science and Technology Education, Faculty of Education, Cukurova University, Adana 01330, \\ Turkey; ogulnaz@cu.edu.tr \\ 3 Department of Horticulture, Faculty of Agriculture, Cukurova University, Adana 01330, Turkey; \\ ebru@cu.edu.tr \\ 4 Department of Park and Garden Plants, Kizilirmak Vocational High School, Cankiri Karatekin University, \\ Cankiri 18100, Turkey; akusvuran@gmail.com \\ 5 Department of Agricultural Machineries and Technologies, Faculty of Agriculture, Cukurova University, \\ Adana 01330, Turkey; hhozturk@cu.edu.tr \\ 6 Department of Agricultural Economics, Faculty of Agriculture, Cukurova University, Adana 01330, Turkey; \\ dbudak@cu.edu.tr \\ * Correspondence: inazli@cu.edu.tr; Tel.: +90-322-338-6084
}

Received: 7 July 2020; Accepted: 1 September 2020; Published: 15 September 2020

check for updates

\begin{abstract}
Sweet sorghum seems to be one of the most promising energy crops for a wide range of ecological conditions thanks to its high yield potential and drought tolerance. A two-year field trial was conducted in a semi-arid Mediterranean environment in Turkey to evaluate the interactive effects of different nitrogen fertilizer levels $\left(100,150\right.$, and $\left.200 \mathrm{~kg} \mathrm{ha}^{-1}\right)$ and humic substances $(0,15,30$, and $45 \mathrm{~L} \mathrm{ha}^{-1}$ ) applications on the biomass and bioethanol yields of sweet sorghum, and combustion characteristics of its bagasse. Sweet sorghum showed a positive response to increased nitrogen fertilizer and humic subtances application levels in terms of biomass and bioethanol production. Lignocellulosic and total bioethanol yields significantly increased up to 2910 and $3336.6 \mathrm{~L} \mathrm{ha}^{-1}$ as a result of the combined application of the $200 \mathrm{~kg} \mathrm{ha}^{-1} \mathrm{~N}$ and $15 \mathrm{~L} \mathrm{ha}^{-1}$ humic subtances (HS), respectively. However, HS application had a detrimental effect on the bagasse combustion quality, because it caused a considerable increase in the mineral and ash concentrations. Briefly, combined application of $200 \mathrm{~kg} \mathrm{ha}^{-1} \mathrm{~N}$ and $15 \mathrm{~L} \mathrm{ha}^{-1}$ HS may be suggested to be applied for the sustainable bioethanol production from sweet sorghum, while the application of HS likely does not provide any economic and environmental benefit if bagasse would be used as solid biofuel feedstock.
\end{abstract}

Keywords: sweet sorghum; bagasse; bioethanol; combustion; nitrogen fertilizer; humic substances

\section{Introduction}

The gradual depletion of fossil fuel reserves and raising concerns about global climate change have brought back interest in biofuels over the last few decades, which have stood out as one of the most promising and environmentally friendly renewable energy sources [1,2]. Bioethanol is currently the most consumed type of biofuel in the global transportation sector, which can be a substitute for gasoline owing to its high octane number, high vaporization heat, and cost-effective 
technology [3-5]. Sweet sorghum is a multifunctional crop that can provide grain, food, feed-fiber syrup, sugar, and bioethanol [6,7]. It has a short growing period (3-5 months), low input requirement, high tolerance to drought, and high biomass production and sugar accumulation (10-20\%) in the stem, which can easily be converted to bioethanol via fermentation $[4,5,8]$. Additionally, sweet sorghum bagasse, the remaining fibrous material after juice extraction, is a valuable by-product that can be used as a feedstock for the production of further lignocellulosic-based bioethanol or heat and power $[9,10]$. Sweet sorghum bagasse contains considerable amounts of carbohydrate polymers (cellulose and hemicellulose), which can be hydrolyzed to fermentable sugars by various conversion technologies, and then fermented to bioethanol [11]. As another option, it can be directly combusted in a bioreactor to generate heat and electricity, thus the energy ratio (output/input) of the bioethanol production may be increased up to 8-10, similar to the sugarcane-based bioethanol industry in Brazil [12]. In both cases, the utilization of the bagasse would increase the energy efficiency of the production chain [13].

The combustion quality of any solid biofuel is mainly determined by ash, moisture, and mineral ( $\mathrm{N}, \mathrm{P}, \mathrm{K}, \mathrm{Ca}, \mathrm{Mg}, \mathrm{S}, \mathrm{Si}$, etc.) concentrations as well as cell wall composition (lignin, cellulose, and hemicellulose). Both high ash and moisture concentrations reduce the heating value, while high ash concentration causes slagging, corrosion, and fouling during combustion [14]. A high concentration of minerals such as $\mathrm{P}, \mathrm{Ca}, \mathrm{K}, \mathrm{Cl}$, and $\mathrm{Si}$ reduces the ash melting point (about $700{ }^{\circ} \mathrm{C}$ ), thus leading to slagging, fouling, and corrosion [15]. When a high concentration of $\mathrm{Si}$ is present in any biomass, alkali metal concentrations react with intrinsic $\mathrm{Si}$ and ash generated from this biomass combustion to turn the ash into a mobile and sticky phase, which causes blockage in the boiler, lead to fouling and hampering furnace mechanisms [16]. On the other hand, high concentration of $\mathrm{N}, \mathrm{S}$, and $\mathrm{Cl}$ contribute to emissions of primary air pollutants [17], Meanwhile, lignin increases the heating value because of its higher carbon and lower oxygen concentration compared with cellulose and hemicellulose $[14,18,19]$.

Nitrogen is often the most limiting nutrient required by sorghum; therefore, it is the most crucial fertilizer that greatly affects the sustainability of sorghum's use in biofuel production [20-22]. Previous studies have exhibited that nitrogen fertilization had a significant effect on the brix or fermentable sugar concentration [23], juice [24], fresh stem [25], bioethanol [26], and biomass yields $[27,28]$ of sweet sorghum. However, $\mathrm{N}$ fertilization constitutes a considerable part of total energy input and production costs in bioenergy production, which must be as low-cost as possible, thus interfering with sustainability [26,29]. Additionally, improper $\mathrm{N}$ management generally results in environmental problems such as nitrous oxide $\left(\mathrm{N}_{2} \mathrm{O}\right)$ emissions and groundwater contamination [30,31]. Therefore, determining the optimal $\mathrm{N}$ fertilizer level is crucial to achieving both economically and environmentally viable biofuel production from sweet sorghum.

In this context, the use of concentrated products of humic substances (humic and fulvic acids) may be a solution to increase bioethanol production and enable the more efficient utilization of applied mineral $\mathrm{N}$ fertilizer in sweet sorghum cultivation $[8,32]$. These are widely recognized as valuable soil amendments, which form a considerable fraction of soil organic matter and abound naturally, especially in aquatic and terrestrial environments [33,34]. Humic substances have positive impacts on crop growth and productivity through improving soil fertility, increasing nutrient uptake and chlorophyll concentration, promoting root length, and stimulating enzymes/hormones, or a combination of several of these processes [8,35-38]. Previous studies have clearly proven that humic substances either significantly increase crop yield and nutrient uptake under adequate mineral nutrition [8,39-41], or maintain crop yield potential even under deficient nutrient supply [42-44].

Although several studies $[25,28,31,45,46]$ examined the juice bioethanol production potential of sweet sorghum under different nitrogen fertilization regimes in various environmental conditions, little is known about the impact of $\mathrm{N}$ fertilization on lignocellulosic bioethanol production from sweet sorghum and combustion quality of its bagasse. Moreover, to our knowledge, there are no previous reports regarding the response of sweet sorghum to the application of humic substances in peer-reviewed literature. Hence, the objective of the present study was to investigate the interactive effects of humic substances and nitrogen fertilization on both juice and lignocellulosic bioethanol 
yield potentials of sweet sorghum and combustion characteristics of its bagasse in a semi-arid Mediterranean environment.

\section{Materials and Methods}

\subsection{Site History and Crop Culture}

The field trial was conducted at the experimental area of the Agricultural Faculty of Cukurova University in Adana ( $37^{\circ} 001 \mathrm{~N}, 35^{\circ} 018 \mathrm{E}$ ), Turkey, during the 2015 and 2016 growing seasons. A typical Mediterranean climate prevails in the region, exhibiting hot and dry summers and temperate and rainy winters. Figure 1 shows the meteorological data.

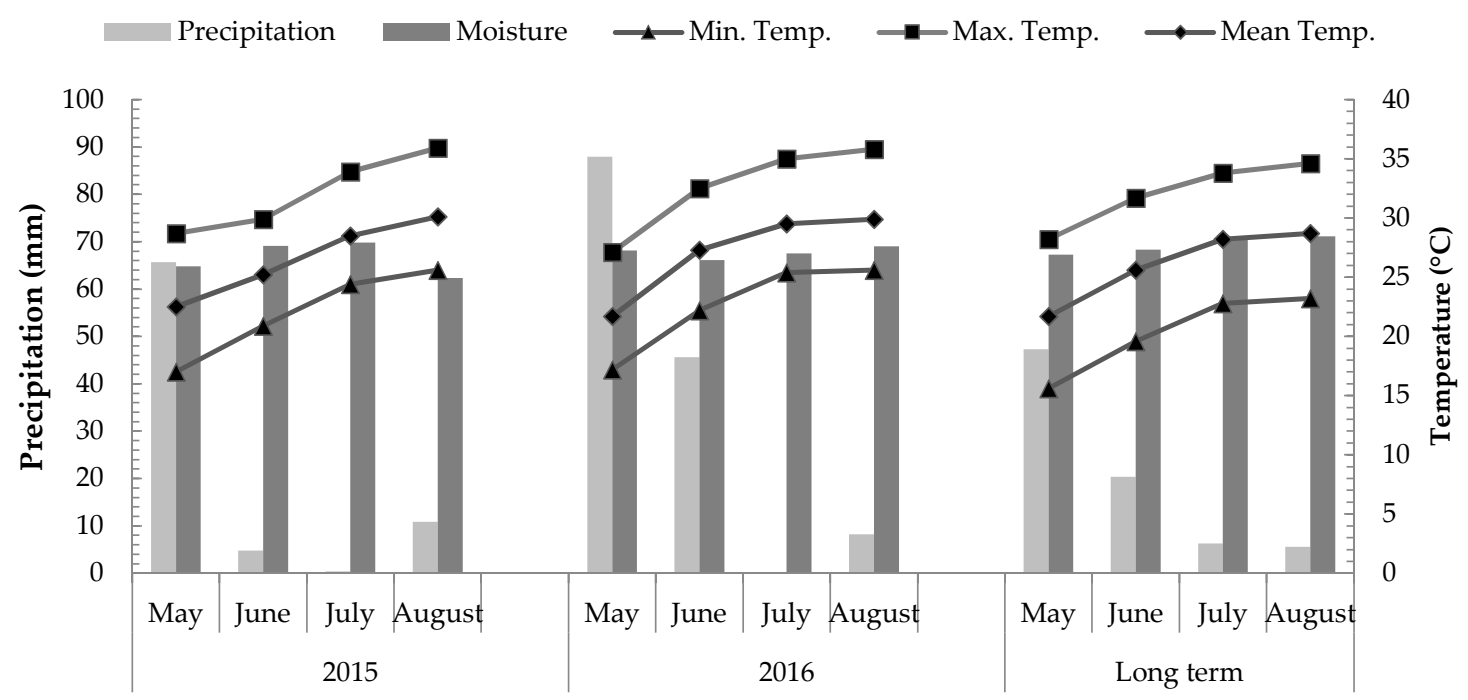

Figure 1. Meteorological data recorded during the field trial and long term (1960-2012). The data were derived from meteorology directorship of Adana, Turkey.

The soil at the site has a loam texture and a low level (0.81\%) of organic matter (Table 1$)$.

Table 1. Initial chemical and physical properties of the soil.

\begin{tabular}{cc}
\hline Property & Depth $\mathbf{( 0 - 3 0 ~ c m )}$ \\
\hline Texture & Loam \\
Organic matter $(\%)$ & 0.81 \\
pH & 8 \\
Salt $\left(\mathrm{mmhos} \mathrm{cm}{ }^{-1}\right)$ & 0.01 \\
Lime $(\%)$ & 11.11 \\
Available P $\left(\mathrm{mg} \mathrm{kg}^{-1}\right)$ & 25 \\
Available K $\left(\mathrm{mg} \mathrm{kg}^{-1}\right)$ & 1992 \\
Available Fe $\left(\mathrm{mg} \mathrm{kg}^{-1}\right)$ & 4.34 \\
Available $\mathrm{Zn}\left(\mathrm{mg} \mathrm{kg}^{-1}\right)$ & 0.14 \\
Available Cu $\left(\mathrm{mg} \mathrm{kg}^{-1}\right)$ & 7.14 \\
Available $\mathrm{Mn}\left(\mathrm{mg} \mathrm{kg}^{-1}\right)$ & 2.14
\end{tabular}

mg: milligram, kg: kilogram, mmhos: milliMhos, cm: centimetre. The soil analyses were conducted in soil and plant analysis laboratory of Agriculture Faculty of Cukurova University.

A non-selective herbicide (glyphosate isopropylamine) was applied nearly a month prior to sowing to kill weeds on the plots. The soil was plowed to approximately $30-40 \mathrm{~cm}$ depth at the beginning of May in both years and then harrowed with field cultivator to form an acceptable seedbed. $\mathrm{P}_{2} \mathrm{O}_{5}$ was distributed at a rate of $100 \mathrm{~kg} \mathrm{ha}^{-1}$ to all plots as a pre-plant fertilizer, but potassium was 
not applied during the study because the soil was rich in K (Table 1). A commercial sweet sorghum cultivar (Gulseker) was used as plant material in the study. The field trial was arranged according to a split-plot design with three replicates considering the $\mathrm{N}$ fertilization levels as the main plots and humic substances applications as sub-plots. The size of each sub-plot was $25.2 \mathrm{~m}^{2}(6 \times 4.2 \mathrm{~m})$. Sweet sorghum seeds were sown in $70 \mathrm{~cm}$ rows with a $10 \mathrm{~cm}$ inter-row spacing at approximately $2-3 \mathrm{~cm}$ depth [27], on 22 May 2015 and 11 May 2016. An insecticide (Lambda-cyhalothrin) was applied twice per growing season to avoid stem borer damage. Irrigation is necessary to grow sweet sorghum in the region owing to the hot and dry summers (Figure 1). In this context, 221 and $208 \mathrm{~mm}$ water was distributed to the plots by means of drip irrigation in the first and second growing seasons, respectively, taking into consideration the soil moisture status at $0-30 \mathrm{~cm}$ depth.

The humic substance (HS) used in this study (TKI-Humas) (5\% organic matter, $12 \%$ humic acid + fulvic acid, 3\% water-soluble potassium oxide, $\mathrm{pH}: 11-13$ ) was produced from leonardite and low-quality lignite by the General Directorate of Turkey Coal Enterprises (TKI) [47]. Four rates of HS (HSO $=$ no HS application, HS15 $=15 \mathrm{~L} \mathrm{ha}^{-1}, \mathrm{HS} 30=30 \mathrm{~L} \mathrm{ha}^{-1}$, and HS45 $=45 \mathrm{~L} \mathrm{ha}^{-1}$ ) were applied to the plots by the drip irrigation system immediately after emergence with a rate of $1 \mathrm{~L}$ per $\mathrm{m}^{3}$ of water [48]. Meanwhile, three rates of $\mathrm{N}$ fertilizer $\left(\mathrm{N} 100=100 \mathrm{~kg} \mathrm{ha}^{-1}\right.$, $\mathrm{N} 150=150 \mathrm{~kg} \mathrm{ha}^{-1}$, and $\mathrm{N} 200=200 \mathrm{~kg} \mathrm{ha}^{-1}$ ) were distributed to the plots according to the method described by Turgut et al. [27] —as 50\% pre-plant and 50\% side dressing when the plants were at the five-leaf stage. Four rows in the center of each plot were harvested and then weighed to determine the fresh biomass weight when the plants had reached the hard dough stage [46].

\subsection{Sample Preparation, Measurements, and Calculations}

Twenty plants were randomly selected from each plot and then divided into two equal parts to be used as both biomass and bioethanol samples. Biomass samples were oven-dried at $60{ }^{\circ} \mathrm{C}$ to a constant weight and finely ground to pass through a $1 \mathrm{~mm}$ screen to determine the dry matter (DM) ratio. Dry matter yield (DMY) was calculated by multiplying the DM ratio and fresh biomass yield.

In the bioethanol samples, the roots, leaves, sheathes, and panicles were removed by hand and then stems were weighed to determine the stem proportion. Thereafter, stems were crushed at least five times to extract the juice through a sugarcane two-roller press. Brix was recorded using a portable digital refractometer (HI 96801, Hanna instruments). Fresh stem yield (FSY), juice yield (JY), sugar concentration (SC), sugar yield (SY), and theoretical juice ethanol yield (JEY) were calculated according to the following equations [49,50]:

$$
\begin{aligned}
& \text { FSY }\left(\mathrm{t} \mathrm{ha}^{-1}\right)=\text { Stem proportion }(\%) \times \text { Fresh biomass yield }\left(\mathrm{t} \mathrm{ha}^{-1}\right) \\
& \text { JY }\left(\mathrm{kg} \mathrm{ha}^{-1}\right)=\mathrm{JR}\left(\text { Juice ratio) }(\%) \times \text { FSY }\left(\mathrm{kg} \mathrm{ha}^{-1}\right)\right. \\
& \text { SC }(\%)=\text { Brix }(\%) \times 0.75 \\
& \text { SY }\left(\mathrm{kg} \mathrm{ha}^{-1}\right)=\mathrm{SC}(\%) \times \mathrm{JY}\left(\mathrm{kg} \mathrm{ha}^{-1}\right)
\end{aligned}
$$

JEY $\left(\mathrm{L} \mathrm{ha}^{-1}\right)=\mathrm{SY}\left(\mathrm{kg} \mathrm{ha}^{-1}\right) \times$ conversion factor $(0.581 \mathrm{~L}$ ethanol per kg sugar $)$

After juice extraction, $1000 \mathrm{~g}$ fresh bagasse samples were oven-dried at $60^{\circ} \mathrm{C}$ to a constant weight and finally ground to pass through a $1 \mathrm{~mm}$ screen to determine bagasse moisture concentration. Dry bagasse yield (DBY) was calculated multiplying by bagasse moisture concentration and fresh bagasse yield.

In order to determine the fiber concentrations of bagasse, the acid detergent fiber (ADF), neutral detergent fiber (NDF), and acid detergent lignin (ADL) concentrations of each sample were first analyzed using the Van Soest [51] method. Thereafter, the lignin, cellulose, and hemicellulose concentrations were determined according to the following equations [52]:

$$
\text { Lignin }=\mathrm{ADL}
$$




$$
\begin{gathered}
\text { Cellulose }=\text { ADF-ADL } \\
\text { Hemiellulose }=\text { NDF-ADF }
\end{gathered}
$$

Theoretical lignocellulosic ethanol yield (LEY) and theoretical total ethanol yield (TEY) were calculated according to the following equations [53,54]:

$$
\begin{aligned}
& \text { LEY }(\mathrm{L})=\text { cellulose }+ \text { hemicellulose concentration }(\%) \\
& \times \text { DBY }\left(\mathrm{t} \mathrm{ha}^{-1}\right) \times 1.11 \times 0.85 \times 0.51 \times 0.85 \times 1000 / 0.79
\end{aligned}
$$

where

1.11 (conversion factor of sugar from cellulose and hemicellulose);

0.85 (process efficiency of sugar from cellulose and hemicellulose);

0.51 (conversion factor of sugar from ethanol);

0.85 (process efficiency of ethanol from sugar);

1000/0.79 (specific gravity of ethanol, $\mathrm{g} \mathrm{ml}^{-1}$ ).

$$
\text { TEY }\left(\mathrm{L} \mathrm{ha}^{-1}\right)=\mathrm{JEY}+\mathrm{LEY}
$$

The bagasse ash concentration $\left(\mathrm{g} \mathrm{kg}^{-1}\right)$ was determined by loss upon ignition in a muffle furnace at $550{ }^{\circ} \mathrm{C}$ for $4 \mathrm{~h} \mathrm{[17].}$

The bagasse $\mathrm{N}$ concentrations $\left(\mathrm{g} \mathrm{kg}^{-1}\right)$ of the samples were analyzed using the Kjeldahl method. The following minerals in bagasse: $\mathrm{P}, \mathrm{K}, \mathrm{Ca}, \mathrm{Mg}$, S, and $\mathrm{Si}\left(\mathrm{g} \mathrm{kg}^{-1}\right)$, were analyzed via inductively coupled plasma optical emission spectrometry (ICP-OES, Perkin Elmer Optima 4300 DV).

The bagasse lower heating value (LHV) was measured using a bomb calorimeter according to the ASTM D5865-04 standard test method.

\subsection{Statistical Analysis}

The data were analyzed according to a split-plot design by an analysis of variance (ANOVA) model using JMP 7.0 (SAS Institute, 1994) statistical software. Significantly different means were separated at a 0.05 probability level using the LSD (Least Significant Difference) test.

\section{Results}

\subsection{Dry Matter Yield and Bioethanol Production}

ANOVA detected that DMY was significantly affected by $\mathrm{N}$ and HS applications, while the interactions $(\mathrm{Y} \times \mathrm{N}, \mathrm{Y} \times \mathrm{HS}, \mathrm{N} \times \mathrm{HS}$, and $\mathrm{Y} \times \mathrm{N} \times \mathrm{HS})$ were never significant for DMY during the study (Table 2). Probability values are presented in supplementary file (Table S1).

Table 2. Results of traits determined by variance analysis. DMY, dry matter yield; FSY, fresh stem yield; JY, juice yield; SY, sugar yield; JEY, juice ethanol yield; DBY, dry bagasse yield; LEY, lignocellulosic ethanol yield; TEY, total ethanol yield; LHV, lower heating value.

\begin{tabular}{cccccccc}
\hline Measurements & $\mathbf{Y}$ & $\mathbf{N}$ & $\mathbf{Y} \times \mathbf{N}$ & $\mathbf{H S}$ & $\mathbf{Y} \times \mathbf{H S}$ & $\mathbf{N} \times \mathbf{H S}$ & $\mathbf{Y} \times \mathbf{N} \times \mathbf{H S}$ \\
\hline DMY & $* *$ & $* *$ & $\mathrm{~ns}$ & $*$ & $\mathrm{~ns}$ & $\mathrm{~ns}$ & $\mathrm{~ns}$ \\
FSY $\left(\mathrm{t} \mathrm{ha}^{-1}\right)$ & $* *$ & $* *$ & $\mathrm{~ns}$ & $* *$ & $\mathrm{~ns}$ & $\mathrm{~ns}$ & $\mathrm{~ns}$ \\
$\mathrm{JR}(\%)$ & $\mathrm{ns}$ & $* *$ & $\mathrm{~ns}$ & $\mathrm{~ns}$ & $\mathrm{~ns}$ & $\mathrm{~ns}$ & $\mathrm{~ns}$ \\
$\mathrm{JY}\left(\mathrm{kg} \mathrm{ha}^{-1}\right)$ & $* *$ & $* *$ & $\mathrm{~ns}$ & $* *$ & $\mathrm{~ns}$ & $\mathrm{~ns}$ & $\mathrm{~ns}$ \\
Brix $(\%)$ & $\mathrm{ns}$ & $\mathrm{ns}$ & $\mathrm{ns}$ & $\mathrm{ns}$ & $\mathrm{ns}$ & $\mathrm{ns}$ & $\mathrm{ns}$ \\
SY $\left(\mathrm{kg} \mathrm{ha}^{-1}\right)$ & $* *$ & $* *$ & $\mathrm{~ns}$ & $* *$ & $\mathrm{~ns}$ & $\mathrm{~ns}$ & $\mathrm{~ns}$ \\
$\mathrm{JEY}\left(\mathrm{L} \mathrm{ha}^{-1}\right)$ & $* *$ & $* *$ & $\mathrm{~ns}$ & $* *$ & $\mathrm{~ns}$ & $\mathrm{~ns}$ & $\mathrm{~ns}$ \\
\hline
\end{tabular}


Table 2. Cont.

\begin{tabular}{|c|c|c|c|c|c|c|c|}
\hline Measurements & $\mathbf{Y}$ & $\mathbf{N}$ & $\mathbf{Y} \times \mathbf{N}$ & HS & $\mathbf{Y} \times \mathbf{H S}$ & $\mathbf{N} \times \mathbf{H S}$ & $\mathbf{Y} \times \mathbf{N} \times \mathbf{H S}$ \\
\hline DBY $\left(\mathrm{t} \mathrm{ha}^{-1}\right)$ & $* *$ & $* *$ & ns & ns & ns & ns & ns \\
\hline Lignin (\%) & $* *$ & ** & $* *$ & $* *$ & $* *$ & $* *$ & $* *$ \\
\hline Cellulose (\%) & ns & $* *$ & $* *$ & $* *$ & $* *$ & $* *$ & $* *$ \\
\hline Hemicellulose (\%) & $* *$ & $* *$ & $* *$ & $* *$ & $* *$ & $* *$ & $* *$ \\
\hline $\operatorname{LEY}\left(\mathrm{L} \mathrm{ha}^{-1}\right)$ & ** & $* *$ & ns & $*$ & ns & ns & $\mathrm{ns}$ \\
\hline TEY $\left(\mathrm{L} \mathrm{ha}^{-1}\right)$ & * & $* *$ & ns & * & ns & ns & ns \\
\hline Bagasse $\mathrm{N}\left(\mathrm{g} \mathrm{kg}^{-1}\right)$ & ** & $* *$ & $* *$ & $* *$ & $* *$ & $* *$ & $* *$ \\
\hline Bagasse $\mathrm{P}\left(\mathrm{g} \mathrm{kg}^{-1}\right)$ & $* *$ & $* *$ & ns & $* *$ & ns & $* *$ & $* *$ \\
\hline Bagasse $\mathrm{K}\left(\mathrm{g} \mathrm{kg}^{-1}\right)$ & $\mathrm{ns}$ & $* *$ & ns & $* *$ & ns & $* *$ & $\mathrm{~ns}$ \\
\hline Bagasse Ca $\left(\mathrm{g} \mathrm{kg}^{-1}\right)$ & ns & ns & ns & ns & ns & ns & ns \\
\hline Bagasse $\mathrm{Mg}\left(\mathrm{g} \mathrm{kg}^{-1}\right)$ & ns & ns & $* *$ & $* *$ & $* *$ & $* *$ & $* *$ \\
\hline Bagasse $S\left(\mathrm{~g} \mathrm{~kg}^{-1}\right)$ & * & * & $* *$ & $* *$ & * & $* *$ & ns \\
\hline Bagasse Si $\left(\mathrm{g} \mathrm{kg}^{-1}\right)$ & * & $* *$ & ns & $* *$ & $* *$ & $* *$ & $\mathrm{~ns}$ \\
\hline Bagasse Moisture (\%) & $* *$ & ns & ns & ns & ns & ns & ns \\
\hline Bagasse Ash (\%) & $\mathrm{ns}$ & $* *$ & ns & $* *$ & ns & $* *$ & ns \\
\hline Bagasse LHV (MJ kg-1) & $\mathrm{ns}$ & $* *$ & $* *$ & $* *$ & $* *$ & $* *$ & $* *$ \\
\hline
\end{tabular}

Averaged across N levels, DMY showed a moderate increase up to N150, while N200 did not create any further significant increase in DMY (Table 3).

Table 3. Effects of the N and HS applications on DMY of sweet sorghum (data over two years).

\begin{tabular}{ccccc}
\hline \multirow{2}{*}{ HS Levels $\left(\mathbf{L ~ h a ~}^{\mathbf{- 1}}\right)$} & \multicolumn{4}{c}{ N Levels $\left.\mathbf{( k g ~ h a}{ }^{-\mathbf{1}}\right)$} \\
\cline { 2 - 5 } & $\mathbf{1 0 0}$ & $\mathbf{1 5 0}$ & $\mathbf{2 0 0}$ & Mean \\
\hline 0 & 7.7 & 10.5 & 10.7 & $9.7^{\mathrm{b}}$ \\
15 & 10.7 & 10.9 & 10.9 & $10.8^{\mathrm{a}}$ \\
30 & 10.6 & 9.8 & 11.3 & $10.6^{\mathrm{ab}}$ \\
45 & 10.2 & 11.5 & 12.2 & $11.3^{\mathrm{a}}$ \\
Mean & $9.8^{\mathrm{b}}$ & $10.7^{\mathrm{a}}$ & $11.3^{\mathrm{a}}$ & \\
\hline
\end{tabular}

${ }^{\mathrm{a}, \mathrm{b}}$ Means followed by the same letters within a column do not differ at $p \leq 0.05$.

The average DMY obtained from the low (HS15), moderate (HS30), and high (HS45) HS applications was $10.8,10.6$, and $11.3 \mathrm{tha}^{-1}$, respectively. These values were higher than the average value $\left(9.7 \mathrm{t} \mathrm{ha}^{-1}\right)$ for HSO. However, there were no significant differences in DMY for HS levels that exceeded HS15.

As with DMY, the interactions never resulted in significance for any of the bioethanol production parameters measured in this study (Table 2). FSY, JY, SY, JEY, LEY, TEY, and bagasse cell-wall components were considerably varied among $\mathrm{N}$ and $\mathrm{HS}$ applications. All these parameters except for hemicellulose concentration were maximized with N200, while there were no significant differences between N150 and N200 in terms of SY and JEY (Table 4). With regard to HS application, HS15 considerably increased FSY, JY, SY, JEY, LEY, TEY, and cellulose and hemicellulose concentrations, while additional levels did not cause any further significant increases in these parameters. Brix was not significantly affected by either $\mathrm{N}$ or HS applications (Table 4). Additionally, $\mathrm{N}$ fertilization led to significant variations in JR and DBY, but they were not clearly influenced by HS application (Table 4). 
Table 4. Effects of the $\mathrm{N}$ and HS applications on bioethanol yield and yield parameters of sweet sorghum (data over two years).

\begin{tabular}{|c|c|c|c|c|c|c|c|c|}
\hline \multirow[t]{2}{*}{ HS Levels (L ha-1) } & \multicolumn{8}{|c|}{ N Levels (kg ha $\left.{ }^{-1}\right)$} \\
\hline & 100 & 150 & 200 & Mean & 100 & 150 & 200 & Mean \\
\hline & \multicolumn{4}{|c|}{ FSY $\left(\mathrm{t} \mathrm{ha}^{-1}\right)$} & \multicolumn{4}{|c|}{ JR (\%) } \\
\hline 0 & 28.4 & 39.7 & 42.3 & $36.8^{\mathrm{b}}$ & 21.43 & 24.80 & 24.41 & 23.55 \\
\hline 15 & 39.3 & 39.9 & 44.5 & $41.2^{\mathrm{a}}$ & 25.93 & 27.59 & 23.81 & 25.77 \\
\hline 30 & 41.0 & 44.1 & 46.9 & $44.0^{\mathrm{a}}$ & 24.14 & 25.30 & 26.43 & 25.29 \\
\hline 45 & 36.8 & 41.9 & 49.2 & $42.6^{\mathrm{a}}$ & 22.38 & 23.77 & 25.68 & 23.94 \\
\hline \multirow[t]{2}{*}{ Mean } & $36.4^{\mathrm{c}}$ & $41.4^{\mathrm{b}}$ & $45.7^{\mathrm{a}}$ & & $23.47^{b}$ & $25.37^{\mathrm{a}}$ & $25.08^{a}$ & \\
\hline & \multicolumn{4}{|c|}{ JY (t ha $\left.{ }^{-1}\right)$} & \multicolumn{4}{|c|}{ Brix $(\%)$} \\
\hline 0 & 6.2 & 10.0 & 10.2 & $8.8^{b}$ & 16.42 & 16.40 & 16.22 & 16.34 \\
\hline 15 & 10.3 & 11.1 & 10.6 & $10.7^{\mathrm{a}}$ & 17.37 & 16.83 & 16.87 & 17.02 \\
\hline 30 & 10.1 & 11.2 & 12.5 & $11.3^{\mathrm{a}}$ & 16.82 & 16.08 & 16.27 & 16.39 \\
\hline 45 & 8.1 & 9.9 & 12.7 & $10.2^{\mathrm{a}}$ & 16.35 & 16.42 & 16.30 & 16.36 \\
\hline \multirow[t]{2}{*}{ Mean } & $8.7^{\mathrm{c}}$ & $10.6^{\mathrm{b}}$ & $11.5^{\mathrm{a}}$ & & 16.74 & 16.43 & 16.41 & \\
\hline & \multicolumn{4}{|c|}{ SY $\left(\mathrm{kg} \mathrm{ha}^{-1}\right)$} & \multicolumn{4}{|c|}{ JEY $\left(\mathrm{L} \mathrm{ha}^{-1}\right)$} \\
\hline 0 & 772.2 & 1220.0 & 1242.5 & $1078.2^{b}$ & 448.7 & 708.8 & 721.9 & $626.4^{b}$ \\
\hline 15 & 1327.7 & 1382.6 & 1334.3 & $1348.2^{\mathrm{a}}$ & 771.4 & 803.3 & 775.2 & $783.3^{\mathrm{a}}$ \\
\hline 30 & 1268.2 & 1353.8 & 1493.6 & $1371.9^{\mathrm{a}}$ & 736.8 & 787.6 & 867.8 & $797.1^{\mathrm{a}}$ \\
\hline 45 & 999.7 & 1215.4 & 1539.2 & $1251.4^{\mathrm{a}}$ & 580.8 & 706.1 & 894.3 & $727.1^{\mathrm{a}}$ \\
\hline \multirow[t]{2}{*}{ Mean } & $1092.0^{\mathrm{b}}$ & $1292.9^{a}$ & $1402.4^{\mathrm{a}}$ & & $634.4^{b}$ & $751.2^{\mathrm{a}}$ & $814.8^{\mathrm{a}}$ & \\
\hline & \multicolumn{4}{|c|}{$\operatorname{DBY}\left(\mathrm{t} \mathrm{ha}^{-1}\right)$} & \multicolumn{4}{|c|}{ Lignin $(\%)$} \\
\hline 0 & 4.7 & 6.7 & 7.0 & 6.1 & $11.15^{\mathrm{k}}$ & $11.66^{\mathrm{i}}$ & $13.12^{b}$ & $11.97^{b}$ \\
\hline 15 & 6.7 & 6.8 & 7.6 & 7.1 & $11.33^{j}$ & $11.86^{h}$ & $12.79^{\mathrm{d}}$ & $11.99^{b}$ \\
\hline 30 & 6.7 & 6.8 & 7.3 & 6.9 & $12.39^{f}$ & $13.41^{\mathrm{a}}$ & $12.92^{c}$ & $12.90^{\mathrm{a}}$ \\
\hline 45 & 6.3 & 6.8 & 7.8 & 6.9 & $10.59^{1}$ & $12.56^{\mathrm{e}}$ & $12.18^{\mathrm{g}}$ & $11.78^{c}$ \\
\hline \multirow[t]{2}{*}{ Mean } & $6.1^{c}$ & $6.8^{b}$ & $7.4^{\mathrm{a}}$ & & $11.36^{c}$ & $12.37^{\mathrm{b}}$ & $12.75^{\mathrm{a}}$ & \\
\hline & \multicolumn{4}{|c|}{ Cellulose (\%) } & \multicolumn{4}{|c|}{ Hemicellulose (\%) } \\
\hline 0 & $42.15^{k}$ & $44.56^{\mathrm{f}}$ & $45.06^{\mathrm{d}}$ & $43.92^{c}$ & $26.41^{\mathrm{e}}$ & $27.02^{b}$ & $24.45^{j}$ & $25.96^{\mathrm{b}}$ \\
\hline 15 & $46.10^{b}$ & $44.42 \mathrm{~g}$ & $47.08^{\mathrm{a}}$ & $45.86^{\mathrm{a}}$ & $25.53^{\mathrm{h}}$ & $27.46^{\mathrm{a}}$ & $26.55^{\mathrm{d}}$ & $26.51^{\mathrm{a}}$ \\
\hline 30 & $44.74^{\mathrm{e}}$ & $46.07^{b}$ & $45.60^{c}$ & $45.47^{b}$ & $24.60^{\mathrm{i}}$ & $24.23^{k}$ & $25.91^{f}$ & $24.91^{\mathrm{d}}$ \\
\hline 45 & $43.06^{j}$ & $43.36^{\mathrm{i}}$ & $43.36^{\mathrm{i}}$ & $43.50^{\mathrm{d}}$ & $25.73^{g}$ & $26.85^{c}$ & $24.25^{k}$ & $25.61^{c}$ \\
\hline \multirow[t]{2}{*}{ Mean } & $44.01^{\mathrm{c}}$ & $44.60^{\mathrm{b}}$ & $45.46^{\mathrm{a}}$ & & $25.57^{b}$ & $26.39^{a}$ & $25.29^{c}$ & \\
\hline & \multicolumn{4}{|c|}{ LEY $\left(\mathrm{L} \mathrm{ha}^{-1}\right)$} & \multicolumn{4}{|c|}{ TEY $\left(\mathrm{L} \mathrm{ha}^{-1}\right)$} \\
\hline 0 & 1650.4 & 2468.8 & 2510.4 & $2209.9^{b}$ & 2099.1 & 3177.6 & 3232.3 & $2836.3^{b}$ \\
\hline 15 & 2501.0 & 2533.4 & 2910.1 & $2648.1^{\mathrm{a}}$ & 3272.4 & 3336.6 & 3685.3 & $3431.4^{\mathrm{a}}$ \\
\hline 30 & 2415.6 & 2459.6 & 2705.8 & $2527.0^{\mathrm{a}}$ & 3152.4 & 3246.2 & 3573.6 & $3324.1^{\mathrm{a}}$ \\
\hline 45 & 2229.5 & 2470.0 & 2743.1 & $2480.9^{a b}$ & 2810.3 & 3176.2 & 3637.3 & $3207.9^{a}$ \\
\hline Mean & $2199.1^{c}$ & $2483.0^{\mathrm{b}}$ & $2717.3^{a}$ & & $2833.5^{c}$ & $3234.1^{b}$ & $3532.1^{\mathrm{a}}$ & \\
\hline
\end{tabular}

\subsection{Bagasse Combustion Characteristics}

According to the variance analysis, the interaction between $\mathrm{N}$ and HS resulted in significance for all of the bagasse combustion characteristics, except for Ca and moisture concentrations (Table 2). Meanwhile, LHV, ash concentration, and all mineral concentrations considered in the study except for Ca were significantly different among HS applications. Mean $\mathrm{N}$ concentration significantly increased with each increment of $\mathrm{N}$ level, while the opposite trend was observed for mean $\mathrm{P}, \mathrm{Mg}$, and $\mathrm{Si}$ concentrations (Table 5). On the other hand, LHV and the concentrations of K, S, and ash were considerably increased by N150, while they exhibited a decreasing trend when the crop was fertilized with N200. 
Table 5. Effects of the $\mathrm{N}$ and HS applications on mineral, moisture, and ash concentrations and cell wall composition of sweet sorghum bagasse (data over two years).

\begin{tabular}{|c|c|c|c|c|c|c|c|c|}
\hline \multirow[t]{2}{*}{ HS Levels $\left(\mathrm{L} \mathrm{ha}^{-1}\right)$} & \multicolumn{8}{|c|}{ N Levels (kg ha $\left.{ }^{-1}\right)$} \\
\hline & 100 & 150 & 200 & Mean & 100 & 150 & 200 & Mean \\
\hline & \multicolumn{4}{|c|}{$\mathrm{N}\left(\mathrm{g} \mathrm{kg}^{-1}\right)$} & \multicolumn{4}{|c|}{$\mathrm{P}\left(\mathrm{g} \mathrm{kg}^{-1}\right)$} \\
\hline 0 & $4.15^{\mathrm{g}}$ & $4.22 \mathrm{fg}$ & $4.75^{\mathrm{c}}$ & $4.37^{\mathrm{c}}$ & $0.55^{\mathrm{c}}$ & $0.60^{\mathrm{b}}$ & $0.50^{\mathrm{d}}$ & $0.55^{b}$ \\
\hline 15 & $4.45^{\mathrm{e}}$ & $4.70^{\mathrm{cd}}$ & $4.90^{b}$ & $4.68^{b}$ & $0.50^{\mathrm{d}}$ & $0.55^{\mathrm{c}}$ & $0.45^{\mathrm{e}}$ & $0.50^{\mathrm{c}}$ \\
\hline 30 & $4.25^{\mathrm{f}}$ & $4.75^{c}$ & $5.05^{\mathrm{a}}$ & $4.68^{b}$ & $0.60^{b}$ & $0.40^{\mathrm{f}}$ & $0.50^{\mathrm{d}}$ & $0.50^{\mathrm{c}}$ \\
\hline 45 & $4.65^{\mathrm{d}}$ & $4.85^{b}$ & $5.05^{\mathrm{a}}$ & $4.85^{\mathrm{a}}$ & $0.65^{\mathrm{a}}$ & $0.60^{b}$ & $0.50^{\mathrm{d}}$ & $0.58^{a}$ \\
\hline \multirow[t]{2}{*}{ Mean } & $4.38^{\mathrm{c}}$ & $4.63^{b}$ & $4.94^{\mathrm{a}}$ & & $0.58^{\mathrm{a}}$ & $0.54^{\mathrm{b}}$ & $0.49^{\mathrm{c}}$ & \\
\hline & \multicolumn{4}{|c|}{$\mathrm{K}\left(\mathrm{g} \mathrm{kg}^{-1}\right)$} & \multicolumn{4}{|c|}{$\mathrm{Ca}\left(\mathrm{g} \mathrm{kg}^{-1}\right)$} \\
\hline 0 & $7.95^{\mathrm{d}}$ & $8.35^{c}$ & $7.55^{\mathrm{e}}$ & $7.95^{c}$ & 1.05 & 1.20 & 1.10 & 1.12 \\
\hline 15 & $8.05^{\mathrm{d}}$ & $8.50^{c}$ & $8.35^{c}$ & $8.30^{b}$ & 1.10 & 1.05 & 1.25 & 1.13 \\
\hline 30 & $9.20^{a b}$ & $9.00^{b}$ & 7.80 de & $8.67^{\mathrm{a}}$ & 1.25 & 1.15 & 1.20 & 1.20 \\
\hline 45 & $8.50^{\mathrm{c}}$ & $9.35^{\mathrm{a}}$ & $8.10^{\mathrm{d}}$ & $8.63^{a}$ & 1.20 & 1.15 & 1.10 & 1.15 \\
\hline \multirow[t]{2}{*}{ Mean } & $8.43^{b}$ & $8.80^{\mathrm{a}}$ & $7.94^{\mathrm{c}}$ & & 1.15 & 1.14 & 1.16 & \\
\hline & \multicolumn{4}{|c|}{$\operatorname{Mg}\left(\mathrm{g} \mathrm{kg}^{-1}\right)$} & \multicolumn{4}{|c|}{$\mathrm{S}\left(\mathrm{g} \mathrm{kg}^{-1}\right)$} \\
\hline 0 & $0.65^{\mathrm{d}}$ & $0.65^{\mathrm{d}}$ & $0.61^{\mathrm{e}}$ & $0.64^{\mathrm{c}}$ & $0.57^{\mathrm{c}}$ & $0.56^{\mathrm{c}}$ & $0.56^{\mathrm{c}}$ & $0.56^{\mathrm{d}}$ \\
\hline 15 & $0.65^{\mathrm{d}}$ & $0.65^{\mathrm{d}}$ & $0.70^{\mathrm{c}}$ & $0.67^{b}$ & $0.56^{\mathrm{c}}$ & $0.57^{\mathrm{c}}$ & $0.57^{\mathrm{c}}$ & $0.57^{\mathrm{c}}$ \\
\hline 30 & $0.75^{b}$ & $0.64^{\mathrm{d}}$ & $0.76^{\mathrm{b}}$ & $0.72^{\mathrm{a}}$ & $0.50^{d}$ & $0.66^{\mathrm{a}}$ & $0.60^{b}$ & $0.59^{b}$ \\
\hline 45 & $0.71^{\mathrm{c}}$ & $0.81^{\mathrm{a}}$ & $0.60^{\mathrm{e}}$ & $0.70^{\mathrm{a}}$ & $0.67^{\mathrm{a}}$ & $0.65^{\mathrm{a}}$ & $0.61^{\mathrm{a}}$ & $0.64^{\mathrm{a}}$ \\
\hline \multirow[t]{2}{*}{ Mean } & 0.69 & 0.69 & 0.67 & & $0.58^{\mathrm{b}}$ & $0.61^{\mathrm{a}}$ & $0.59^{b}$ & \\
\hline & \multicolumn{4}{|c|}{$\mathrm{Si}\left(\mathrm{g} \mathrm{kg}^{-1}\right)$} & \multicolumn{4}{|c|}{ Ash $\left(\mathrm{g} \mathrm{kg}^{-1}\right)$} \\
\hline 0 & $5.45^{\mathrm{d}}$ & $5.45^{\mathrm{d}}$ & $5.65^{\mathrm{d}}$ & $5.52^{b}$ & $18.16^{\mathrm{ij}}$ & $20.50^{\mathrm{fg}}$ & $\begin{array}{c}19.37 \\
\text { gh }\end{array}$ & $19.34^{\mathrm{d}}$ \\
\hline 15 & $6.45^{\mathrm{c}}$ & $6.55^{c}$ & $6.65^{c}$ & $6.55^{\mathrm{a}}$ & $19.30 \mathrm{hi}$ & 22.50 de & $21.36^{\text {ef }}$ & $21.06^{\mathrm{c}}$ \\
\hline 30 & $7.85^{\mathrm{a}}$ & $6.40^{c}$ & $5.40^{\mathrm{c}}$ & $6.55^{\mathrm{a}}$ & $27.03^{b}$ & $23.36^{\mathrm{cd}}$ & $18.06^{\mathrm{j}}$ & $22.82^{b}$ \\
\hline 45 & $6.60^{\mathrm{c}}$ & $7.20^{b}$ & $5.40^{\mathrm{d}}$ & $6.40^{\mathrm{a}}$ & $24.07^{b}$ & $28.70^{\mathrm{a}}$ & 19.00 hij & $23.92^{\mathrm{a}}$ \\
\hline \multirow[t]{2}{*}{ Mean } & $6.59^{\mathrm{a}}$ & $6.40^{b}$ & $5.78^{c}$ & & $22.14^{\mathrm{b}}$ & $23.77^{\mathrm{a}}$ & $19.45^{\mathrm{c}}$ & \\
\hline & \multicolumn{4}{|c|}{ Moisture (\%) } & \multicolumn{4}{|c|}{ LHV (MJ kg $\left.{ }^{-1}\right)$} \\
\hline 0 & 71.02 & 72.27 & 71.96 & 71.75 & $17.96^{\mathrm{d}}$ & $17.89^{\mathrm{e}}$ & $18.10^{\mathrm{c}}$ & $17.98^{\mathrm{a}}$ \\
\hline 15 & 71.17 & 71.16 & 71.11 & 71.14 & $17.38^{\mathrm{i}}$ & $18.19^{\mathrm{a}}$ & $18.13^{b}$ & $17.90^{b}$ \\
\hline 30 & 71.50 & 72.12 & 72.01 & 71.88 & $17.95^{\mathrm{d}}$ & $17.60^{\mathrm{h}}$ & $17.85^{\mathrm{f}}$ & $17.80^{\mathrm{c}}$ \\
\hline 45 & 70.44 & 72.24 & 70.76 & 71.15 & $17.74^{\mathrm{g}}$ & $17.84^{\mathrm{f}}$ & $17.38^{\mathrm{i}}$ & $17.65^{d}$ \\
\hline Mean & 71.03 & 71.95 & 71.46 & & $17.76^{\mathrm{b}}$ & $17.88^{\mathrm{a}}$ & $17.87^{\mathrm{a}}$ & \\
\hline
\end{tabular}

${ }^{a-j}$ Means followed by the same letters within a column do not differ at $p \leq 0.05$.

Averaged across HS levels, HS45 had the significantly highest values for N, P, S, and ash concentrations, but the lowest value for LHV. In contrast, the highest LHV and the lowest concentrations of $\mathrm{N}, \mathrm{K}, \mathrm{Ca}, \mathrm{Mg}$, $\mathrm{Si}$, and ash were obtained from HSO.

On the other hand, moisture concentration was not significantly affected by either $\mathrm{N}$ or HS applications.

\section{Discussion}

$\mathrm{N}$ fertilization may be considered one of the main factors affecting the bioethanol and biomass yield potentials of sweet sorghum [26-28,31,45]. Our results confirm that increasing $N$ fertilization significantly affected the DMY and bioethanol production parameters of sweet sorghum except for brix in the present study. Sweet sorghum positively responded to $\mathrm{N}$ fertilization, achieving the significantly highest FSY, JY, DBY, LEY, TEY, and bagasse cellulose concentration when the crop was fertilized with N200. Additionally, the crop also exhibited positive, but comparatively limited responses to N fertilization with respect to DMY, SY, and JEY, attaining the significant increases up to N150. In contrast, the majority of earlier studies conducted in a wide range of ecological conditions generally reported that either a comparatively low $\mathrm{N}$ input was sufficient for the optimization of biomass and bioethanol 
production or that $\mathrm{N}$ fertilization had no evident effect on the crop's DMY (Table 6), probably owing to its capability to extract relatively high quantities of soil $\mathrm{N}$ under $\mathrm{N}$-limiting conditions [28].

Table 6. Optimization of dry matter and bioethanol yields in diverse ecological conditions under different $\mathrm{N}$ fertilization regimes.

\begin{tabular}{|c|c|c|c|c|c|}
\hline Field Experiment & Region & $\begin{array}{l}\text { N Levels } \\
\left(\text { kg ha }^{-1}\right)\end{array}$ & Parameters & $\begin{array}{c}\text { Optimum N Level } \\
\left(\mathrm{kg} \mathrm{ha}^{-1}\right)\end{array}$ & Optimum Yield \\
\hline \multirow[t]{4}{*}{ Maw et al. [26] } & Midwestern USA & $0,56,112,168,224$ & SY & 112 & $1217 \mathrm{~kg} \mathrm{ha}^{-1}$ \\
\hline & & & JY & 112 & $10,294 \mathrm{~kg} \mathrm{ha}^{-1}$ \\
\hline & & & JEY & 112 & $692 \mathrm{~L} \mathrm{ha}^{-1}$ \\
\hline & & & DBY & 112 & $17.6 \mathrm{tha}^{-1}$ \\
\hline \multirow[t]{2}{*}{ Singh et al. [20] } & Uttarakhand, India & $0,60,120,180$ & SY & 120 & $2130 \mathrm{~kg} \mathrm{ha}^{-1}$ \\
\hline & & & JEY & 120 & 6461.11 $\mathrm{L} \mathrm{ha}^{-1}$ \\
\hline \multirow[t]{2}{*}{ Kurai et al. [55] } & Patancheru, India & $0,63,90,150$ & SY & 90 & $5300 \mathrm{~kg} \mathrm{ha}^{-1}$ \\
\hline & & & JEY & 90 & $2900 \mathrm{~L} \mathrm{ha}^{-1}$ \\
\hline Thivierge et al. [46] & Quebec, Canada & $0,40,80,120,160$ & JEY & 80 & 865-1539 $\mathrm{L} \mathrm{ha}^{-1}$ \\
\hline \multirow[t]{2}{*}{ Tang et al. [31] } & Northern China & $0,60,120,240$ & JEY & 120 & $3089 \mathrm{~L} \mathrm{ha}^{-1}$ \\
\hline & & & DMY & 60 & $8.6 \mathrm{tha}^{-1}$ \\
\hline Erickson et al. [56] & Florida, USA & $45,90,135,180$ & DMY & 45 & $15 \mathrm{tha}^{-1}$ \\
\hline Cosentino et al. [57] & Southern Italy & $0,60,120,180$ & DMY & 0 & $17.9 \mathrm{tha}^{-1}$ \\
\hline
\end{tabular}

For example, Maw et al. [26] tested four N fertilization levels (N0, N56, N112, N168, and N224) in the Midwestern USA and concluded that the three-year means of SY, JY, JEY, and DBY were considerably increased by N fertilization levels from N0 to N112, while LEY and TEY significantly increased up to N168. Singh et al. [20] reported that both SY and JEY significantly increased up to N120 and then sharply decreased with N180 in Uttarakhand, India. In a semi-arid climate, N90 was found to be sufficient to achieve optimal results for each of SY, DMY, and JEY [55]. Additionally, it was found that a low N input of N80 considerably increased the JEY, but no significant improvement was observed for $\mathrm{N}$ fertilization levels exceeding N80 in the ecozones (Mixedwood Plains and Boreal Shield) of Canada [46]. Tang et al. [31] tested four N fertilization levels (N0, N60, N120, and N240) in Northern China and demonstrated that N60 provided a marked increase in DMY compared with N0, while JEY significantly increased up to N120. Other studies showed that sweet sorghum exhibited no evident DMY in response to N fertilization levels ranging from N45 to N180 in Florida, USA [56] and N0 to N180 in Southern Italy [57]. These results also suggest that the optimal N fertilization level required for satisfactory dry matter and bioethanol yields of sweet sorghum is highly dependent on the prevailing environmental conditions in the specific agro-ecosystem. The amounts of organic matter and plant-available forms of $\mathrm{N}$ (i.e., $\mathrm{NO}_{3}$ and $\mathrm{NH}_{4}$ ) in soil stand out as the essential environmental factors that determine crop needs for $\mathrm{N}$ fertilizer [58-60]. Soil plant-available $\mathrm{N}$ concentration is augmented by organic matter mineralization and nitrification, which are influenced by several biotic and abiotic factors such as soil organic matter concentration, microbial activity, $\mathrm{pH}$, moisture, aerial, and temperature [61]. Prasad and Power [62] stated that soils in cooler areas generally have much more organic matter than those in warmer areas; therefore, the presence of plant-available $\mathrm{N}$ can exceed $200 \mathrm{~kg} \mathrm{ha}^{-1}$ in a growing season. In the experiment location, soils are identified as poor in organic matter and plant-available $\mathrm{N}$ concentration $[63,64]$, thus requiring relatively high $\mathrm{N}$ input to achieve satisfactory crop yield potentials $[44,65,66]$. This suggestion may be a possible reason sweet sorghum requires greater $\mathrm{N}$ input at the location than in different environments. An earlier study [27], which reported that sweet sorghum achieved an optimal DMY with N100 among five N fertilization levels (N0, N50, N100, N150, and N200) in a cooler area (Northwestern Turkey) that has a higher soil organic matter (2.4\%), supports this suggestion. Likewise, Kizil et al. [65] indicated that sorghum-sudangrass hybrid grown at the experiment location needed N180 for optimal DMY, which is comparatively higher than those (N60) reported in cooler locations [67] that have relatively high soil organic matter $(1.68-2.15 \%)$. 
Several previous studies focused on the $\mathrm{N}$ fertilization effects on cell-wall composition pointed out that the biosynthesis of both lignin and cellulose was down-regulated under high nitrogen input, thus increasing lodging and reducing stem mechanical strength and disease resistance [68-71]. Our results conflict with this inference because increasing the $\mathrm{N}$ fertilization levels stimulated all cell-wall components of sweet sorghum bagasse in the present study. Previous studies confirmed [72,73] our results in that $\mathrm{N}$ fertilization positively affected either the lignin or both the lignin and cellulose concentrations of forage or sweet sorghum. However, our results contrast with those reported in these studies in which $\mathrm{N}$ fertilization generally induced a reduction in the crop's hemicellulose concentration. These results suggest that environmental conditions may effectively determine the cell wall composition response of sorghum to $\mathrm{N}$ fertilization.

As we observed with $\mathrm{N}$ fertilization, sweet sorghum positively responded to HS application with respect to FSY, JY, SY, JEY, LEY, and TEY, attaining significant increases up to HS15. The increases that occurred by means of HS15 application were recorded as nearly 12\% in both DMY and FSY, $22 \%$ in JY, $25 \%$ in both SY and JEY, $20 \%$ in LEY, and lastly $17 \%$ in TEY. These substantial increases might be associated with the beneficial effects of humic substances on crop growth and productivity, which are categorized as either indirect or direct $[8,74]$. Indirect effects involve the improvement of the soil's physical, chemical, and biological conditions [8,75], while direct effects are attributed to various biochemical regulations in plant tissues such as the increase in nutrient uptake, $\mathrm{CO}_{2}$ adsorption, chlorophyll concentration and membrane permeability, modification in metabolite concentration, maintenance of the levels of vitamins and amino acids, and the acceleration of the respiration process and hormonal growth responses [8,75,76].

As mentioned in the result section, both $\mathrm{N}$ and HS applications had no clear effect on the brix of sweet sorghum. With regards to the $\mathrm{N}$ fertilization effect on brix, our results coincide with those reported by Maw et al. [26] Kumar et al. [77], and Uchino et al. [78]; however, they are in conflict with those reported by Mekdad and El- Sharif [23], Sawargaonkar et al. [25], and Holou and Stevens [79]. Regarding HS effects on brix, Akin [47] observed that the HS source used in the study did not cause any significant variation in the sugar concentration of grape. In contrast, several previous studies [76,80-82] suggested that HS application had a positive effect on the brix or sugar concentration of different crops, perhaps because it induced some changes in the activities of enzymes involved in carbohydrate metabolism [80]. Regarding the $\mathrm{N}$ fertilization effects on combustion characteristics, moderate (N150) or high (N200) $\mathrm{N}$ fertilization levels induced significant increases in mean $\mathrm{N}, \mathrm{K}$, and $\mathrm{S}$ concentrations, while the opposite was found in mean $\mathrm{P}, \mathrm{Mg}$, and Si concentrations. Similarly, Erickson et al. [56] suggested that $\mathrm{N}$ concentrations of sweet sorghum bagasse tended to increase with each increment of the $\mathrm{N}$ fertilization level from N45 to N180, while the opposite trend was generally observed for $\mathrm{P}$ concentration in Florida, USA. Meanwhile, significant reduction in Si concentration caused by increasing $\mathrm{N}$ fertilization levels may be attributed to a combination of the following factors. Excessive $\mathrm{N}$ fertilization may lead to a depletion in the amount of Si that is available to plants, thus reducing Si uptake in the plant's roots [83], and the plants generally accumulate more Si under N-deficient conditions to withstand biotic and abiotic stresses [84]. These results are supported by previous studies that proved that $\mathrm{N}$ fertilization significantly reduced the Si concentration of several bioenergy crops under Mediterranean climatic conditions [15,85].

However, HS application positively affected all mineral concentrations of sweet sorghum bagasse measured in the study. Mineral concentration response to HS application was greater at the high (HS45) and moderate (HS30) levels than that at the low level (HS15). HS application increased N, $\mathrm{K}, \mathrm{Mg}$, and $\mathrm{Si}$ concentrations with each $\mathrm{N}$ fertilization level. HS levels enhanced $\mathrm{N}$ concentration up to $12 \%, 15 \%$, and $6 \%$; $\mathrm{K}$ concentration up to $16 \%, 12 \%$, and $7 \%$; Mg concentration up to $9 \%, 25 \%$, and $25 \%$; S concentration up to $18 \%, 18 \%$, and $\% 9$; and Si concentration up to $44 \%, 32 \%$, and $18 \%$ in the plots fertilized with N100, N150, and N200, respectively. However, the HS levels increased P concentration up to $18 \%$ in plots fertilized with N100, while they did not provide any clear effect on $\mathrm{P}$ concentration of bagasse in the plots fertilized with N150 and N200. These results may suggest that 
HS had a limited effect on $\mathrm{P}$ uptake of sweet sorghum under moderate and high $\mathrm{N}$ input conditions. HS must be considered as a soil amendment rather than fertilizer because it contains either none or a very low amount of nutrients [75]. Therefore, the increase in the mineral concentration of sweet sorghum bagasse after HS application is principally associated with the stimulatory effects of HS on nutrient solubility and availability. HS increases the solubility of minerals by building metal complexes and chelating agents $[33,75,86]$, enhancing the availability of nutrient elements by holding them on the mineral surfaces, and converting them into forms that are available to plants $[35,37,87,88]$, which often results in the increased mineral concentration of plants, particularly when these minerals are scarce in the soil $[89,90]$. Similar to what we detected in sweet sorghum, previous studies have clearly exhibited that HS increased the concentration of $\mathrm{N}$ and $\mathrm{K}$ in grain sorghum [91]; N, P, K, Ca, and $\mathrm{Mg}$ in maize [92] and wheat [93]; N, P, K, and Ca in sugarcane [81]; N, P, K, and S in canola [33]; and lastly only Si concentration in soybean [94].

$\mathrm{N}$ and $\mathrm{S}$ are the mineral elements that contribute to NOx and SOx emissions in a combustion system, so their concentrations in biomass should not exceed 6 and $1 \mathrm{~g} \mathrm{~kg}^{-1}$ in dry matter, respectively [14,95]. In the present study, we found that the crop always exhibited $\mathrm{N}$ and $\mathrm{S}$ concentrations below the threshold.

As with Brix, $\mathrm{N}$ or HS applications had no evident effect on moisture concentration of sweet sorghum bagasse in the study. Our results are consistent with those reported by Holou and Stevens [79], in which $\mathrm{N}$ fertilization did not distinctly affect water concentration of sweet sorghum bagasse in Midwest USA. To store any biomass for a long time without molding, the moisture concentration should not exceed $25 \%$ at time of harvest [96]. In the present study, we observed that the moisture concentration of the crop was quite higher than the threshold, indicating that the bagasse must be dried to be stored safely for a long time.

Both $\mathrm{N}$ and HS applications affected the ash concentration of sweet sorghum bagasse. Mean ash concentration was significantly increased by N150, then significantly reduced when the crop was fertilized with N200. In contrast, ash concentration tended to considerably increase with each increment of HS level. HS application increased ash concentration by up to $48 \%, 40 \%$, and $10 \%$ in the plots fertilized with N100, N150, and N200, respectively. Ash concentration results achieved in the study are probably associated with changes in the ash-forming mineral concentrations $(\mathrm{P}, \mathrm{K}, \mathrm{Ca}, \mathrm{Cl}, \mathrm{Mg}, \mathrm{Si}, \mathrm{Al}$, $\mathrm{Na}$, and so on) [97] caused by $\mathrm{N}$ and HS applications.

With regards to LHV, N150 significantly increased the mean LHV of the bagasse, while N200 did not provide any further significant increases in mean LHV. However, mean LHV exhibited a significantly decreasing trend with increased HS level. At each $\mathrm{N}$ fertilization level, the LHV was always decreased by moderate and high HS applications. The LHV of solid biofuels is mainly determined by the carbon, hydrogen, oxygen, and volatile concentrations of any biomass $[14,98]$. However, ash and lignin concentrations are considered additional indicators of LHV. Every $1 \%$ increase in the ash concentration leads to a decrease in the LHV of a fuel by $0.2 \mathrm{MJ} \mathrm{kg}^{-1}$ [99]. Moreover, a high lignin concentration favors LHV owing to its lower oxygen concentration $\left(<300 \mathrm{~g} \mathrm{~kg}^{-1}\right)$ compared with cellulose and hemicellulose $\left(>500 \mathrm{~g} \mathrm{~kg}^{-1}\right)$, which dramatically decreases the calorific value $[14,18,19]$. Hence, high lignin, but low ash concentration is desirable for high LHV in thermochemical conversion processes. Consequently, variation in the LHV of sweet sorghum bagasse observed in this study may correlate with changes in the concentrations of all or several LHV indicators $(\mathrm{C}, \mathrm{H}, \mathrm{O}$, volatile, ash, and lignin) caused by $\mathrm{N}$ and $\mathrm{HS}$ applications.

\section{Conclusions}

$\mathrm{N}$ fertilization strongly affected bioethanol yield parameters in the study, except for brix. Although N150 optimized JEY, the significantly highest LEY and TEY were achieved with N200, mainly owing to the fact that DBY and cellulose concentration exhibited a significant increasing trend with increased $\mathrm{N}$ level. Besides, N200 significantly increased only N concentration in sweet sorghum bagasse, while it produced $\mathrm{LHV}, \mathrm{P}, \mathrm{K}, \mathrm{Ca}, \mathrm{Mg}, \mathrm{S}, \mathrm{Si}$, and ash concentrations that were similar to or lower than those for N100 and N150. 
On the other hand, sweet sorghum positively responded to HS application, achieving the optimized JEY, LEY, and TEY with HS15. However, HS application had a detrimental effect on the combustion quality of sweet sorghum bagasse, because it induced generally significant increases in mineral and ash concentrations. Additionally, HS levels had no clear effect on DBY, but considerably reduced LHV, indicating that HS application probably had no any positive effect on energy yield of sweet sorghum bagasse.

The results suggest that combined application of $200 \mathrm{~kg} \mathrm{ha}^{-1} \mathrm{~N}$ and $15 \mathrm{~L} \mathrm{ha}^{-1} \mathrm{HS}$ seemed to be favorable for the sustainable whole crop bioethanol production from sweet sorghum, while the application of HS probably does not provide any economic and environmental benefit if combustion is a target after juice ethanol production in a semi-arid Mediterranean environment. However, as the response of sweet sorghum to combined application of $\mathrm{N}$ fertilizer and bio-stimulants considerably changes depending on the cultivars and soil types, determination of economic optimum application level in diverse sites of Mediterranean should be main focus in future studies to enable sustainable bioethanol production from sweet sorghum cultivars.

Supplementary Materials: The following are available online at http://www.mdpi.com/2073-4395/10/9/1397/s1, Table S1. Probability values $(p>0.05)$ determined by variance analysis; Abbreviations.

Author Contributions: Conceptualization, R.I.N., E.K., and V.T.; methodology, R.I.N., O.G., V.T., and A.K., software, A.K., D.B.B., V.T., and H.H.O.; formal analysis, R.I.N., V.T., A.K., H.H.O., and D.B.B.; investigation, R.I.N., O.G., and E.K.; resources, R.I.N., O.G., and E.K.; data curation, R.I.N. and A.K., writing—original draft preparation, R.I.N., O.G., and V.T.; writing-review and editing, R.I.N., H.H.O., E.K., D.B.B., and V.T.; visualization, R.I.N., A.K., O.G., and V.T.; supervision, R.I.N. and V.T.; project administration, R.I.N. and V.T.; funding acquisition, V.T. All authors have read and agreed to the published version of the manuscript.

Funding: This research was funded by Scientific Research Project Unit of Cukurova University (BAP), Adana, Turkey under project no. FOA-2015-3722.

Conflicts of Interest: The authors declare no conflict of interest.

\section{References}

1. Zegada-Lizarazu, W.; Monti, A. Are we ready to cultivate sweet sorghum as a bioenergy feedstock? A review on field management practices. Biomass Bioenergy 2012, 40, 1-12. [CrossRef]

2. Da Silva, T.M.; de Oliveira, A.B.; de Moura, J.G.; da Trindade Lessa, B.F.; de Oliveira, L.S.B. Potential of Sweet Sorghum Juice as a Source of Ethanol for Semi-arid Regions: Cultivars and Spacing Arrangement Effects. Sugar Tech 2019, 21, 145-152. [CrossRef]

3. Gnansounou, E.; Dauriat, A.; Wyman, C.E. Refining sweet sorghum to ethanol and sugar: Economic trade-offs in the context of North China. Bioresour. Technol. 2005, 96, 985-1002. [CrossRef] [PubMed]

4. Cifuentes, R.; Bressani, R.; Rolz, C. The potential of sweet sorghum as a source of ethanol and protein. Energy Sustain. Dev. 2014, 21, 13-19. [CrossRef]

5. Dar, R.A.; Dar, E.A.; Kaur, A.; Phutela, U.G. Sweet sorghum-a promising alternative feedstock for biofuel production. Renew. Sustain. Energy Rev. 2018, 82, 4070-4090.

6. Doggett, H. Sorghum, 2nd ed.; Tropical Agricultural Series; Longman Scientific: Essex, UK, 1988.

7. Habyarimana, E.; Lorenzoni, C.; Laureti, D.; Di Fonzo, N. Biomass production and drought resistance at the seedling stage and in field conditions in sorghum. Maydica [Sorghum bicolor L.]. Maydica 2002, 47, 305-309. (In Italian)

8. Rao, M.M.; Govindasamy, R.; Chandrasekaran, S. Effect of humic acid on Sorghum vulgare var. CSH-9. Curr. Sci. 1987, 56, 1273-1276.

9. Reddy, B.V.S.; Rao, P.S.; Kumar, A.A.; Reddy, P.S.; Rao, P.P.; Sharma, K.K.; Blummel, M.; Reddy, C.R. Sweet sorghum as a biofuel crop where are we now. In Proceedings of the 6th International Biofuels Conference; ICRISAT: Hyderabad, India, 2009; pp. 193-202.

10. Singh, M.P.; Erickson, J.E.; Sollenberger, L.E.; Woodard, K.R.; Vendramini, J.M.B.; Fedenko, J.R. Mineral composition and biomass partitioning of sweet sorghum grown for bioenergy in the southeastern USA. Biomass Bioenergy 2012, 47, 1-8. [CrossRef] 
11. Goshadrou, A.; Karimi, K.; Taherzadeh, M.J. Bioethanol production from sweet sorghum bagasse by Mucor hiemalis. Ind. Crops Prod. 2011, 34, 1219-1225. [CrossRef]

12. Goldemberg, J.; Guardabassi, P. The potential for first-generation ethanol production from sugarcane. Biofuels Bioprod. Biorefin. Innov. Sustain. Econ. 2010, 4, 17-24. [CrossRef]

13. Monti, A.; Venturi, G. Comparison of the energy performance of fibre sorghum, sweet sorghum and wheat monocultures in northern Italy. Eur. J. Agron. 2003, 19, 35-43. [CrossRef]

14. Lewandowski, I.; Kicherer, A. Combustion quality of biomass: Practical relevance and experiments to modify the biomass quality of Miscanthus $\times$ giganteus. Eur. J. Agron. 1997, 6, 163-177. [CrossRef]

15. Nassi o Di Nasso, N.; Angelini, L.G.; Bonari, E. Influence of fertilization and harvest time on fuel quality of giant reed (Arundo donax L.) in Central Italy. Eur. J. Agron. 2010, 32, 219-227. [CrossRef]

16. Hossain, N.; Zaini, J.; Mahlia, T.M.I.; Azad, A.K. Elemental, morphological and thermal analysis of mixed microalgae species from drain water. Renew. Energy 2019, 131, 617-624. [CrossRef]

17. Iqbal, Y.; Lewandowski, I. Inter-annual variation in biomass combustion quality traits over five years in fifteen Miscanthus genotypes in south Germany. Fuel Process. Technol. 2014, 121, 47-55. [CrossRef]

18. Demirbas, A. Calculation of higher heating values of biomass fuels. Fuel 1997, 76, 431-434. [CrossRef]

19. Friedl, A.; Padouvas, E.; Rotter, H.; Varmuza, K. Prediction of heating values of biomass fuel from elemental composition. Anal. Chim. Acta 2005, 544, 191-198. [CrossRef]

20. Singh, S.P.; Joshi, Y.P.; Singh, R.K.; Meena, V.S. Influence of nitrogen levels and seed rate on growth, yield and quality of sweet sorghum. Ann. Biol. 2014, 30, 89-93.

21. Reddy, P.S.; Reddy, B.V.; Ashok Kumar, A.; Rao, P.S. Standardization of nitrogen fertilizer rate for sugar yield optimization in sweet sorghum. J. SAT Agric. Res. 2008, 6, 1-4.

22. Holou, R.A.; Stevens, G. Impact of nitrogen fertilization and the soil type on the quality and yield of sweet sorghum juice. In Proceedings of the XVI International Plant Nutrition Colloquium, Sacramento, CA, USA, 26-30 August 2009.

23. Mekdad, A.A.A.; El-Sherif, A.M.A. The Effect of Nitrogen and Potassium Fertilizers on Yield and Quality of Sweet Sorghum Varieties under Arid Regions Conditions. Int. J. Curr. Microbiol. Appl. Sci. 2016, 5, 811-823. [CrossRef]

24. Tomar, G.S.; Sai, S. Effect of planting density and levels of nitrogen on ethanol production of sweet sorghum (Sorghum bicolor [L.] Moench) varieties. Pharma Innov. J. 2018, 7, 4-7.

25. Sawargaonkar, G.L.; Patil, M.D.; Wani, S.P.; Pavani, E.; Reddy, B.V.S.R.; Marimuthu, S. Nitrogen response and water use efficiency of sweet sorghum cultivars. Field Crop. Res. 2013, 149, 245-251. [CrossRef]

26. Maw, M.J.; Houx, J.H., III; Fritschi, F.B. Sweet sorghum ethanol yield component response to nitrogen fertilization. Ind. Crops Prod. 2016, 84, 43-49. [CrossRef]

27. Turgut, I.; Bilgili, U.; Duman, A.; Acikgoz, E. Production of sweet sorghum (Sorghum bicolor L. Moench) increases with increased plant densities and nitrogen fertilizer levels. Acta Agric. Scand. Sect. B Soil Plant 2005, 55, 236-240.

28. Adams, C.B.; Erickson, J.E.; Singh, M.P. Investigation and synthesis of sweet sorghum crop responses to nitrogen and potassium fertilization. Field Crop. Res. 2015, 178, 1-7. [CrossRef]

29. Amaducci, S.; Monti, A.; Venturi, G. Non-structural carbohydrates and fibre components in sweet and fibre sorghum as affected by low and normal input techniques. Ind. Crops Prod. 2004, 20, 111-118. [CrossRef]

30. Sowiński, J.; Głą, L. The effect of nitrogen fertilization management on yield and nitrate contents in sorghum biomass and bagasse. Field Crop. Res. 2018, 227, 132-143. [CrossRef]

31. Tang, C.; Yang, X.; Chen, X.; Ameen, A.; Xie, G. Sorghum biomass and quality and soil nitrogen balance response to nitrogen rate on semiarid marginal land. Field Crop. Res. 2018, 215, 12-22. [CrossRef]

32. Verlinden, G.; Coussens, T.; De Vliegher, A.; Baert, G.; Haesaert, G. Effect of humic substances on nutrient uptake by herbage and on production and nutritive value of herbage from sown grass pastures. Grass Forage Sci. 2010, 65, 133-144. [CrossRef]

33. Akinremi, O.O.; Janzen, H.H.; Lemke, R.L.; Larney, F.J. Response of canola, wheat and green beans to leonardite additions. Can. J. Soil Sci. 2000, 80, 437-443. [CrossRef]

34. Hamad, M.M.; Tantawy, M.F. Effect of different Humic Acids Sources on the Plant Growth, Cal-cium and Iron Utilization by Sorghum. Egypt. J. Soil Sci. 2018, 58, 291-307.

35. Stevenson, F.J. Humus Chemistry: Genesis, Composition, Reactions, 2nd ed.; John Wiley and Sons, Inc.: Hoboken, NJ, USA, 1994. 
36. Canellas, L.P.; Olivares, F.L.; Facanha-Okorokova, A.L.; Facanha, A.R. Humic acids isolated from earthworm compost enhance root elongation, lateral root emergence, and plasma membrane $\mathrm{H}$-ATPase activity in maize roorts. Plant Physiol. 2002, 30, 1951-1957. [CrossRef] [PubMed]

37. Akinci, S.; Buyukkeskin, T.; Eroglu, A.; Erdogan, B.E. The Effect of Humic Acid on Nutrient Composition in Broad Bean (Vicia faba L.). Roots Not. Sci. Biol. 2009, 1, 81-87. [CrossRef]

38. Abdel-Mawgoud, A.M.R.; El-Greadly, N.H.M.; Helmy, Y.I.; Singer, S.M. Responses of tomato plants to different rates of humic-based fertilizer and NPK fertilization. J. Appl. Sci. Res. 2007, 3, 169-174.

39. Moghadam, H.R.T.; Khamene, M.K.; Zahedi, H. Effect of humic acid foliar application on growth and quantity of corn in irrigation withholding at different growth stages. Maydica 2014, 59, 124-128.

40. Ece, A.; Saltali, K.; Eryigit, N.; Uysal, F. The effects of leonardite applications on climbing bean (Phaseolus vulgaris L.) yield and soil properties. J. Agron. 2007, 6, 480-483.

41. Demir, M.; Noyan, O.F.; Oğuz, I. Leonardit kullanımı ile birlikte azaltılmış azotlu gübre uygulamalarının bitki verim ve toprak özellikleri üzerine etkileri. Sak. Ünive. Fen Edeb. Derg. 2012, 1, 445-455.

42. Selim, E.M.; El-Neklawy, A.S.; El-Ashry, S.M. Beneficial effects of humic substances on soil fertility to fertigated potato grown on sandy soil. Libyan Agric. Res. Cent. J. Int. 2010, 1, 255-262.

43. Magdi, T.A.; Selim, E.M.; El-Ghamry, A.M. Integrated effects of bio and mineral fertilizers and humic substances on growth, yield and nutrient contents of fertigated cowpea (Vigna unguiculata L.) grown on sandy soils. J. Agron. 2011, 10, 34-39.

44. Nazli, R.I.; Kusvuran, A.; Inal, I.; Demirbas, A.; Tansi, V. Effects of different organic materials on forage yield and quality of silage maize (Zea mays L.). Turk. J. Agric. For. 2014, 38, 23-31. [CrossRef]

45. Almodares, A.; Hadi, M.R.; Ranjbar, M.; Taheri, R. The effects of nitrogen treatments, cultivars and harvest stages on stalk yield and sugar content in sweet sorghum. Asian J. Plant Sci. 2007, 6, 423-426.

46. Thivierge, M.N.; Chantigny, M.H.; Bélanger, G.; Seguin, P.; Bertrand, A.; Vanasse, A. Response to nitrogen of sweet pearl millet and sweet sorghum grown for ethanol in eastern Canada. Bioenergy Res. 2015, 8, 807-820. [CrossRef]

47. Akin, A. The Effects of Some Summer Pruning and Humic Substance Applications on the Nutritional Value of 'Alphonse Lavallée' Grape Cultivar. Erwerbs-Obstbau 2018, 60, 271-274. [CrossRef]

48. Gezgin, S.; Dursun, N.; Yilmaz, G. Bitki Yetiştiriciliğinde humik ve fulvik asit kaynağı olan Tki-humas kullanımı. Sak. Üniv. Fen Edeb. Derg. 2012, 1, 159-163.

49. Wortmann, C.S.; Liska, A.J.; Ferguson, R.B.; Lyon, D.J.; Klein, R.N.; Dweikat, I. Dryland performance of sweet sorghum and grain crops for biofuel in Nebraska. Agron. J. 2010, 102, 319-326. [CrossRef]

50. Teetor, V.H.; Duclos, D.V.; Wittenberg, E.T.; Young, K.M.; Chawhuaymak, J.; Riley, M.R.; Ray, D.T. Effects of planting date on sugar and ethanol yield of sweet sorghum grown in Arizona. Ind. Crops Prod. 2011, 34, 1293-1300. [CrossRef]

51. Van Soest, P.J. Use of detergents in the analysis of fibrous feeds. 2. A rapid method for the determination of fiber and lignin. J. Assoc. Off. Agric. Chem. 1963, 46, 829-835.

52. Hodgson, E.M.; Lister, S.J.; Bridgwater, A.V.; Clifton-Brown, J.; Donnison, I.S. Genotypic and environmentally derived variation in the cell wall composition of Miscanthus in relation to its use as a biomass feedstock. Biomass Bioenergy 2010, 34, 652-660. [CrossRef]

53. Institution of Japan Energy (Ed.) Biomass Handbook; Hua, Z.Z., Shi, Z.P., Translators; Chemistry Industry Press: Beijing, China, 2006; pp. 166-167, 172.

54. Zhao, Y.L.; Dolat, A.; Steinberger, Y.; Wang, X.; Osman, A.; Xie, G.H. Biomass yield and changes in chemical composition of sweet sorghum cultivars grown for biofuel. Field Crop. Res. 2009, 111, 55-64. [CrossRef]

55. Kurai, T.; Morey, S.R.; Wani, S.P.; Watanabe, T. Efficient rates of nitrogenous fertiliser for irrigated sweet sorghum cultivation during the post-rainy season in the semi-arid tropics. Eur. J. Agron. 2015, 71, 63-72. [CrossRef]

56. Erickson, J.E.; Woodard, K.R.; Sollenberger, L.E. Optimizing sweet sorghum production for biofuel in the southeastern USA through nitrogen fertilization and top removal. Bioenergy Res. 2012, 5, 86-94. [CrossRef]

57. Cosentino, S.L.; Mantineo, M.; Testa, G. Water and nitrogen balance of sweet sorghum (Sorghum bicolor moench (L.)) cv. Keller under semi-arid conditions. Ind. Crops Prod. 2012, 36, 329-342. [CrossRef]

58. Carpenter-Boggs, L.; Pikul, J.L.; Vigil, M.F.; Riedell, W.E. Soil nitrogen mineralization influenced by crop rotation and nitrogen fertilization. Soil Sci. Soc. Am. J. 2000, 64, 2038-2045. [CrossRef] 
59. Villaseñor, D.; Zagal, E.; Stolpe, N.; Hirzel, J. Relationship between mineralized nitrogen during anaerobic incubations and residual effect of nitrogen fertilization in two rice paddy soils in Chile. Chil. J. Agric. Res. 2015, 75, 98-104. [CrossRef]

60. Cai, A.; Xu, H.; Shao, X.; Zhu, P.; Zhang, W.; Xu, M.; Murphy, D.V. Carbon and nitrogen mineralization in relation to soil particle-size fractions after 32 years of chemical and manure application in a continuous maize cropping system. PLoS ONE 2016, 11, e0152521. [CrossRef]

61. Barker, A.V.; Bryson, G.M. Section II, Essential elements-macronutrients. In Handbook of Plant Nutrition; Barker, A.V., Pilbeam, D.J., Eds.; CRC Press: Boca Raton, FL, USA, 2007; pp. 21-43.

62. Prasad, R.; Power, J.F. Section VIII, Nitrogen, in Soil Fertility Management for Sustainable Agriculture; CRC Press: Boca Raton, FL, USA, 1997; pp. 115-169.

63. Koca. Potential Use of Preplant Soil Mineral Nitrogen Content in First Crop Corn Fertilization Programe in Cukurova Region. Master's Dissertation, Cukurova University, Adana, Turkey, 2013.

64. Pamiralan, H.; Gok, M. Bakla Vejetasyonu Altında Nitrifikasyon İnhibitörü Uygulamasının Nodülasyon, Nodül, Kök ve Kök Üstünde Toplam Azot ve Toprakta Nmin Değerlerine Etkisi. Sdü Ziraat Fakültesi Derg. 2018, 3, 519-527.

65. Kizil, S.; Tansi, V.; Avci, M. An investigation on determining the most suitable nitrogen and zinc fertilizer doses for the main crops of Sorghum-Sudangrass Hybrids(Sorghum bicolor $\times$ Sorghum sudanense) in the Cukurova Region (Turkey). Biol. Divers. Conserv. 2010, 3, 117-122.

66. Ozkan, A.; Ulger, A.C. Çukurova Ekolojik Koşullarında Değişik Azot Dozu Uygulamalarının İki Cin Mısırı (Zea mays L. everta Sturt.) Çeşidinde Tane Verimi ve Bazı Tarımsal Özelliklere Etkisi. Yüzüncü Yıl Üniv. Tarım Bilimleri Derg. 2011, 21, 198-208.

67. Iptas, S.; Brohi, A.R. Effect of nitrogen rate and stubble height on dry matter yield, crude protein content and crude protein yield of a sorghum-sudangrass hybrid [Sorghum bicolor (L.) Moench $\times$ Sorghum sudanense (Piper) Stapf.] in the three-cutting system. J. Agron. Crop Sci. 2003, 189, 227-232. [CrossRef]

68. Matsuyama, N. The effect of ample nitrogen fertilizer on cell-wall materials and its significance to rice blast disease. Jpn. J. Phytopathol. 1975, 41, 56-61. [CrossRef]

69. Wang, T.; Park, Y.B.; Cosgrove, D.J.; Hong, M. Cellulose-pectin spatial contacts are inherent to never-dried Arabidopsis primary cell walls: Evidence from solid-state nuclear magnetic resonance. Plant Physiol. 2015, 168, 871-884. [CrossRef] [PubMed]

70. Zhang, W.; Wu, L.; Ding, Y.; Yao, X.; Wu, X.; Weng, F.; Li, G.; Liu, Z.; Tang, S.; Ding, C.; et al. Nitrogen fertilizer application affects lodging resistance by altering secondary cell wall synthesis in japonica rice (Oryza sativa). J. Plant Res. 2017, 130, 859-871. [CrossRef] [PubMed]

71. Ogden, M.; Hoefgen, R.; Roessner, U.; Persson, S.; Khan, G. Feeding the Walls: How Does Nutrient Availability Regulate Cell Wall Composition? Int. J. Mol. Sci. 2018, 19, 2691. [CrossRef] [PubMed]

72. Blummel, M.; Zerbini, E.; Reddy, B.V.S.; Hash, C.T.; Bidinger, F.; Ravi, D. Improving the production and utilization of sorghum and pearl millet as livestock feed: Methodological problems and possible solutions. Field Crop. Res. 2003, 84, 123-142. [CrossRef]

73. Sher, A.; Hassan, F.U.; Ali, H.; Hussain, M.; Sattar, A. Enhancing forage quality through appropriate nitrogen dose, seed rate and harvest stage, in sorghum cultivars grown in Pakistan. Grassl. Sci. 2017, 63, 15-22. [CrossRef]

74. Chen, Y.; Aviad, T. Effect of humic substances on plant growth. In Humic Substances in Soil and Crop Sciences: Selected Readings; MacCarthy, P., Ed.; American Society of Agronomy: Madison, WI, USA, 1990; pp. 161-186.

75. Tan, K.H. Section VIII, Agronomic importance of humic matter. In Humic Matter in Soil and the Environment: Principles and Controversies; CRC Press: Boca Raton, FL, USA, 2003.

76. Ahmed, A.H.; Nesiem, M.R.; Hewedy, A.M.; Sallam, H. Effect of some simulative compounds on growth, yield and chemical composition of snap bean plants grown under calcareous soil conditions. J. Am. Sci. 2010, $6,552-569$.

77. Kumar, S.R.; Shrotria, P.K.; Deshmukh, J.P. Characterizing nutrient management effect on yield of sweet sorghum genotypes. World J. Agric. Sci. 2008, 4, 787-789.

78. Uchino, H.; Watanabe, T.; Ramu, K.; Sahrawat, K.L.; Marimuthu, S.; Wani, S.P.; Ito, O. Effects of nitrogen application on sweet sorghum (Sorghum bicolor (L.) Moench) in the semi-arid tropical zone of India. Jpn. Agric. Res. Q. JARQ 2013, 47, 65-73. [CrossRef] 
79. Holou, R.A.; Stevens, G. Juice, sugar, and bagasse response of sweet sorghum (Sorghum bicolor (L.) Moench cv. M81E) to N fertilization and soil type. GCB Bioenergy 2012, 4, 302-310. [CrossRef]

80. Merlo, L.; Ghisi, R.; Passera, C.; Rascio, N. Effects of humic substances on carbohydrate metabolism of maize leaves. Can. J. Plant Sci. 1991, 71, 419-425. [CrossRef]

81. Govindasmy, R.; Chandrasekaran, S. Effect of humic acids on the growth, yield and nutrient content of sugarcane. Sci. Total Environ. 1992, 117, 575-581. [CrossRef]

82. Khan, A.; Khan, M.Z.; Hussain, F.; Akhtar, M.E.; Gurmani, A.R.; Khan, S. Effect of humic acid on the growth, yield, nutrient composition, photosynthetic pigment and total sugar contents of peas (Pisum sativum L.). J. Chem. Soc. Pak. 2013, 35, 206-211.

83. Ma, J.F.; Yamaji, N. Silicon uptake and accumulation in higher plants. Trends Plant Sci. 2006, 11, 392-397. [CrossRef] [PubMed]

84. Marschner, H. Mineral Nutrition of Higher Plants, 2nd ed.; Academic Press: London, UK, 1995.

85. Nazli, R.I.; Tansi, V. Influences of nitrogen fertilization and harvest time on combustion quality of four perennial grasses in a semi-arid Mediterranean climate. Ind. Crops Prod. 2019, 128, 239-247. [CrossRef]

86. Chen, Y.; De Nobili, M.; Aviad, T. Section IV. Stimulatory effects of humic substances on plant growth. In Soil Organic Matter in Sustainable Agriculture; Magdoff, F., Weil, R.R., Eds.; CRC Press: Boca Raton, FL, USA, 2004; pp. 103-129.

87. Tipping, E. Cation Binding by Humic Substances; Cambridge University Press: Cambridge, UK, 2002 ; Volume 12.

88. Kulikova, N.A.; Stepanova, E.V.; Koroleva, O.V. Mitigating activity of humic substances: Direct influence on biota. In Use of Humic Substances to Remediate Polluted Environments: From Theory to Practice; Springer: Dordrecht, The Netherlands, 2005; pp. 285-309.

89. Garcia, A.C.; Santos, L.A.; de Souza, L.G.A.; Tavares, O.C.H.; Zonta, E.; Gomes, E.T.M.; Mina, J.M.G.; Berbara, R.L.L. Vermicompost humic acids modulate the accumulation and metabolism of ROS in rice plants. J. Plant Physiol. 2016, 192, 56-63. [CrossRef]

90. Shah, Z.H.; Rehman, H.M.; Akhtar, T.; Alsamadany, H.; Hamooh, B.T.; Mujtaba, T.; Daur, I.; Al Zahrani, Y.; Alzahrani, H.A.S.; Shawkat, A.; et al. Humic substances: Determining potential molecular regulatory processes in plants. Front. Plant Sci. 2018, 9, 263. [CrossRef]

91. Afifi, M.M.I.; El-Sayed, G.A.M.; Manal, A.; El-Gamal, H.; Massoud, O.N. Synergistic effect of biofertilizers containing N-fixer, $\mathrm{P}$ and $\mathrm{K}$ solubilizers and humic substances on Sorghum bicolor productivity. Middle East J. Appl. Sci. 2014, 4, 1065-1074.

92. Khaled, H.; Fawy, H.A. Effect of different levels of humic acids on the nutrient content, plant growth, and soil properties under conditions of salinity. Soil Water Res. 2011, 6, 21-29. [CrossRef]

93. Katkat, A.V.; Çelik, H.; Turan, M.A.; Asik, B.B. Effects of soil and foliar applications of humic substances on dry weight and mineral nutrients uptake of wheat under calcareous soil conditions. Aust. J. Basic Appl. Sci. 2009, 3, 1266-1273.

94. Rashad, R.T.; Hussien, R.A. Studying the Solubility, Availability, and Uptake of Silicon (Si) from Some Ore Minerals in Sandy Soil. Sains Tanah J. Soil Sci. Agroclimatol. 2018, 15, 69-82. [CrossRef]

95. Prochnow, A.; Heiermann, M.; Plöchl, M.; Amon, T.; Hobbs, P.J. Bioenergy from permanent grassland-A review: 2. Combustion. Bioresour. Technol. 2009, 100, 4945-4954. [CrossRef] [PubMed]

96. Lewandowski, I.; Clifton-Brown, J.C.; Scurlock, J.M.O.; Huisman, W. Miscanthus: European experience with a novel energy crop. Biomass Bioenergy 2000, 19, 209-227. [CrossRef]

97. Vassilev, S.V.; Vassileva, C.G.; Song, Y.C.; Li, W.Y.; Feng, J. Ash contents and ash-forming elements of biomass and their significance for solid biofuel combustion. Fuel 2017, 208, 377-409. [CrossRef]

98. Obernberger, I.; Brunner, T.; Barnthaler, G. Chemical properties of solid biofuels-significance and impact. Biomass Bioenergy 2006, 30, 973-982. [CrossRef]

99. Jenkins, B.; Baxter, L.L.; Miles, T.R. Combustion properties of biomass. Fuel Proc. Technol. 1998, 54, 17-46. [CrossRef]

(C) 2020 by the authors. Licensee MDPI, Basel, Switzerland. This article is an open access article distributed under the terms and conditions of the Creative Commons Attribution (CC BY) license (http://creativecommons.org/licenses/by/4.0/). 